\title{
Alessandro Galante Garrone, historien de la Révolution française
}

Anna-Maria Rao

\section{(2) OpenEdition \\ 12 Journals}

Édition électronique

URL : https://journals.openedition.org/ahrf/6513

DOI : 10.4000/ahrf.6513

ISSN : 1952-403X

\section{Éditeur :}

Armand Colin, Société des études robespierristes

\section{Édition imprimée}

Date de publication : 1 juin 2006

Pagination : 219-237

ISSN : 0003-4436

\section{Référence électronique}

Anna-Maria Rao, «Alessandro Galante Garrone, historien de la Révolution française », Annales historiques de la Révolution française [En ligne], 344 | avril-juin 2006, mis en ligne le 01 juin 2009, consulté le 23 avril 2022. URL : http://journals.openedition.org/ahrf/6513 ; DOI : https://doi.org/ 10.4000/ahrf.6513

Ce document a été généré automatiquement le 23 avril 2022.

Tous droits réservés 


\title{
Alessandro Galante Garrone, historien de la Révolution française
}

\author{
Anna-Maria Rao
}

En Italie, la presse, les revues historiques, les académies, ont déjà rendu hommage à Alessandro Galante Garrone (1909-2003), rappelant sa biographie culturelle et politique, et ses ouvrages ${ }^{1}$. Il avait tracé lui-même son itinéraire politique et intellectuel dans sa Conversation sur liberté et démocratie avec Paolo Borgna, publiée en 1994 sous le titre Il mite giacobino. Dans ce petit livre savoureux il ironisait sur l'étiquette de jacobin qu'on lui avait collée et qui, plus généralement, était volontiers employée pour critiquer ceux qui avaient fait partie du mouvement antifasciste Justice et liberté et du Parti d'actiondont il avait été un des fondateurs - , trop souvent considérés comme élitistes et minoritaires, d'esprit abstrait et moraliste. Il racontait avoir été longuement persécuté par cette réputation "jacobine», d'avoir été fréquemment étiqueté de "jacobin truculent ", jusqu'à ce que le quotidien Il Giorno, en 1974, ait publié une entrevue avec lui sous le titre Il mite giacobino non s'arrende, qu'on pourrait traduire par Le jacobin débonnaire ne se rend pas. Nommé par quelques-uns le «bon jacobin piémontais », ou un "jacobin sui generis », considéré par d'autres "trop jacobin », cette habitude invétérée l'avait conduit à se poser la question du comment et pourquoi les hommes politiques du $\mathrm{XIX}^{\mathrm{e}}$ siècle s'étaient lancés cette épithète à la tête. Il concluait avec humour qu'il voulait bien accepter ce titre, à condition de spécifier qu'en aucun cas il n'aurait fait partie «de la race des jacobins qui coupaient les têtes, mais de celle des jacobins auxquels on coupait la tête $»^{2}$.

2 Au cours de cette conversation de 1994, il avait parcouru la suite des attentes, des illusions, des déceptions des hommes qui s'étaient battus contre le fascisme, les avancées mais aussi les échecs traversés par la nouvelle Italie républicaine. La démocratie et la liberté étaient des objectifs à conquérir et à réaliser au jour le jour ; la liberté n'était pas une valeur figée, mais « pour se maintenir en tant que telle, elle doit savoir se faire libératrice ${ }^{3}$. Il puisait la formule de la " liberté libératrice » chez Adolfo Omodeo et, comme celui-ci, il aimait la répéter souvent, pour souligner que «l'histoire 
pose toujours aux hommes, au-delà et contre la consolidation d'un état politique et social, de nouveaux problèmes concrets de libération $»^{4}$.

3 Avant d'être historien et professeur d'histoire, il avait choisi en 1933 d'entrer dans la magistrature, parce que c'était la seule fonction publique accessible à ceux qui ne voulaient pas s'inscrire au parti fasciste ${ }^{5}$. Il ne s'agissait donc pas d'une véritable vocation, mais il poursuivit cette carrière avec scrupule et, grâce à l'enseignement de Piero Calamandrei, il avait ressenti toute « l'élévation, même terrible, de la fonction [...] choisie ", et comme il écrira à son ami Franzo Grande Stevens, "un sens presque religieux de consternation " face à la nécessité du jugement, qui l'avait convaincu «qu'il n'était pas si grave d'avoir dû renoncer à m'appliquer à l'étude de l'histoire, comme j'avais rêvé $»^{6}$. Collaborateur du quotidien La Stampa de Turin à partir de 1955, il pourra enfin réaliser son rêve après 1963 et se dédier entièrement à l'histoire et à son enseignement, devenu professeur d'histoire moderne dans la Faculté de Droit de l'Université de Turin et par la suite professeur d'histoire du Risorgimento dans la Faculté de Lettres de la même Université, après trois ans d'enseignement à Cagliari.

4 Adonné aux études historiques dès les années 1930, c'est surtout dans les années 1940 qu'il commença ses recherches sur la Révolution française. En avril 1952 il sera élu membre du Comité de la Société des Études robespierristes - Kohatiro Takahashi était présent à la séance - à la place de Caron, décédé7, et il y restera jusqu'à sa mort. Ce sont justement ses rapports scientifiques avec la France et les Annales historiques de la Révolution française que je voudrais évoquer ici ${ }^{8}$.

Certes, on connaît bien en France son Gilbert Romme, publié en Italie en 1959 avec une préface de Georges Lefebvre, traduit en français seulement en 1971, avec la même préface $^{9}$. À l'occasion du colloque de Riom des 19 et 20 mai 1995, dont les actes furent dédiés à Alessandro Galante Garrone et à la mémoire d'Albert Soboul ${ }^{10}$, Jean Ehrard expliquait ainsi la dédicace et la présidence d'honneur confiée à l'auteur de l'Histoire d'un révolutionnaire:

« comment ne pas souligner ce que la mémoire de Romme doit, paradoxalement, à un pays, l'Italie, à peu près étranger à sa propre culture ? À l'origine de ce fécond paradoxe, un homme et un livre : tout au long des exposés, le second a été, comme il se devait, l'ouvrage de référence, et le climat du colloque n'aurait pas eu la même chaleur sans la personnalité généreuse du premier. En remettant par les mains de son maire à Alessandro Galante Garrone la médaille de la ville, Riom a justement reconnu ce que lui devait la patrie de Gilbert Romme $»^{11}$.

6 Quant à l'auteur du livre, à l'époque âgé de quatre-vingt-six ans, le condensé de ses propos publiés dans les actes nous livrent deux constats : le souvenir de son "grand ami » Franco Venturi, «à la suggestion duquel il avait entrepris ses propres recherches»; et les points essentiels qu'il avait trouvés dans la pensée et le comportement politique de Romme, à savoir, "le culte de la loi » - une véritable «religion de la loi » - et «la place centrale de l'idée d'égalité $»^{12}$. Son livre qui faisait autorité et qui avait déjà fourni au colloque de Riom de 1965 le soutien de sa reconstruction et de ses pièces justificatives, ne l'empêcha pas trente ans plus tard, comme le rappelle Michel Vovelle, d'inviter les historiens à « ensevelir son œuvre sous notre nouvelle érudition $»^{13}$. Du reste, lui-même n'avait jamais considéré terminées ses recherches sur le sujet, en dépit du caractère imposant des documents déjà accumulés. $\mathrm{Au}$ colloque de Riom de 1965 il avait communiqué son plaisir d'avoir pu combler des lacunes dans les procès-verbaux de la Société des Amis de la loi fondée par Romme au début de 1790, grâce à un renseignement de Renzo De Felice; ou bien d'avoir pu se 
procurer, "tout récemment ", une photocopie du texte complet du Journal de Gilbert Romme qui lui permettait de signaler un fragment inédit par rapport à l'édition qu'en avait publiée Marc De Vissac ${ }^{14}$.

On retrouve là deux caractéristiques essentielles du travail d'historien de Galante Garrone, jamais abandonné des années 1930 à sa mort: le lien fondamental entre histoire, historiographie et politique ; l'inlassable recherche de documents, même sur des sujets déjà longuement abordés et abondamment défrichés, soulignant avec modestie le caractère toujours provisoire de la recherche historique, travail en continuel progrès.

C'est le cas de Romme, sur lequel on reviendra. C'est le cas aussi de l'autre grand chantier de recherches de sa vie, les études sur Filippo Buonarroti, entreprises dans les années 1940 (après une première incursion dès 1931); ou encore de ses recherches sur Quinet, abordées pendant les mêmes années. Autour de ces recherches, beaucoup d'amis qui l'inspiraient et le poussaient dans sa voie par leurs suggestions et même par leurs observations critiques: Franco Venturi, Adolfo Omodeo et d'autres en Italie, Georges Lefebvre, Albert Soboul, Jacques Godechot et d'autres en France.

À côté du Romme, son livre sur Filippo Buonarroti e i rivoluzionari dell'Ottocento est aussi bien connu en France grâce à la traduction française parue en 1975, vingt-quatre ans après la première édition italienne, mais seulement trois ans après la deuxième édition ${ }^{15}$. Le dédiant à Piero Calamandrei, il remerciait Albert Soboul, auquel il ajoutait, dans la Note à la deuxième édition, les noms d'Armando Saitta, Pia Onnis Rosa, Jacques Godechot, Arthur Lehning, et, surtout, Georges Lefebvre, «à qui je dois tant, pour d'autres travaux aussi $»^{16}$. Une première version de ce livre, sous le titre Gli ultimi anni di Filippo Buonarroti (1828-1837) avait déjà paru en 1949, et avait obtenu le "Prix Gramsci » pour les études historiques. Mais il l'avait remanié, en considération aussi de la publication du Buonarroti d'Armando Saitta ${ }^{17}$. Et déjà en 1948 il avait publié à Turin son Buonarroti et Babeuf ${ }^{18}$. Il y reviendra encore, toute sa vie. En 1953 il publie dans la revue Movimento operaio, "Filippo Buonarroti e i Convenzionali in esilio ", tirant profit des papiers de Vadier conservés à Toulouse dont Jacques Godechot, qu'il remerciait, lui avait procuré le microfilm ${ }^{19}$. Il remerciait aussi l'ami Richard Cobb qui l'avait aidé à déchiffrer l'écriture du vieux Vadier, tandis qu'à son tour il communiquait la copie des lettres de Barère trouvées parmi ces documents à Leo Gershoy, qui préparait « une biographie de Barère très bien documentée $»^{20}$. Tout un monde d'amitiés, de solidarités et d'échanges scientifiques se dessine dans ces notes. Et le tiré à part conservé dans la Bibliothèque Soboul à Vizille porte la dédicace autographe suivante : «À Marius le plus ami des historiens le plus historien des amis. Sandro ».

Son intérêt pour Filippo Buonarroti, le premier "révolutionnaire de profession", comme l'avait défini Elisabeth L. Eisenstein ${ }^{21}$, ainsi que pour Romme et pour Babeuf c'est-à-dire pour la « religion de la loi » et pour l'égalité, pour l'exil et pour le problème de l'unité du mouvement révolutionnaire - reste constant : il suffit de rappeler l'article "De Prairial à la Conspiration des Égaux " publié dans le volume d'hommages à Franco Venturi de 1985 (où il annonçait pourtant son «congé de ses études bien aimées sur Romme et sur Buonarroti $»^{22}$ ), l'article rédigé pour le dictionnaire italien des interprétations de la Révolution française de $1989^{23}$ et l'édition, en 1992, avec Venturi encore, de La Riforma dell'Alcorano de Buonarroti ${ }^{24}$. Encore une fois, de nouveaux documents interviennent qui intègrent, confirment, nuancent, parfois corrigent ce qui était déjà connu : dans l'article de 1985, c'est le tour de deux fragments d'une défense 
inédite, la dernière, de Gilbert Romme, datée du 29 prairial, le jour même de sa condamnation, dont la photocopie lui avait été envoyée en 1983 par Jacques Girard qui les avait achetés avec d'autres documents que Marc de Vissac devait avoir connus ${ }^{25}$.

11 Dans ce même article, il rappelait avec d'autres dettes scientifiques qu'il avait accumulées au fil des années depuis son Gilbert Romme (les documents sur l'entourage de Tissot et de Buonarroti que lui avait procurés son ami Sylvain Goujon, le descendant d'un frère du conventionnel qu'il avait connu dans les années 1960) la genèse de ses études sur Buonarroti : «Si mon premier écrit sur Buonarroti remonte au lointain 1948, ma curiosité pour ce personnage naquit bien avant, dès les années universitaires (en 1931 !), quand je tombai sur son opuscule alors inconnu, et signé Camillo, dont je fis signe dans un petit écrit de 1934 ». Et il ajoutait ironiquement entre parenthèses : «Je le dis pour démentir une chercheuse américaine qui pensait que mon intérêt pour Buonarroti était né après la guerre, pour d'évidentes sympathies [...] politiques ». Il devait surtout à Franco Venturi, expliquait-il, de l'avoir poussé à cultiver son intérêt pour le révolutionnaire toscan : Venturi, qui s'en était occupé à Paris dans les années 1930, «le lendemain de la Résistance, que nous avions vécue ensemble, voulut généreusement me donner les fruits de ses premières recherches. Il fut ainsi que l'auteur du présent écrit, qui était alors juge à Turin, s'improvisa historien ${ }^{26}$.

12 Le point de départ de sa recherche avait été la considération historiographique de la Révolution élaborée et modifiée au fil des circonstances par Buonarroti dès le lendemain de Thermidor et qui avait abouti en 1828 à sa Conspiration pour l'égalité dite de Babeuf: une considération, écrivait-il, enrichie mais aussi devenue mythique et que Mathiez avait reprise et admirée, "avec quelque discutable dilatation", pour son "hyperbolique exaltation de Robespierre $»^{27}$. Ce qui surtout l'intéressait c'était le lien entre histoire et politique dans Buonarroti, chez qui il soulignait le caractère embryonnaire, rudimentaire, de la réflexion historiographique, surchargée par le poids de la mémoire personnelle et surtout par l'intention pratique de susciter de nouvelles énergies révolutionnaires. Ce même lien le frappait chez Quinet dont la Révolution était un « cri de bataille, un appel de l'exilé politique aux Français de son temps », beaucoup plus qu'un ouvrage historiographique fondé sur des recherches et sur des documents : d'où son succès immédiat et, ensuite, son oubli, jusqu'à ce que François Furet ne l'ait situé, à côté de Tocqueville et de Michelet, parmi les trois historiens majeurs du XIX ${ }^{\mathrm{e}}$ siècle de la Révolution française ce qui, critiquait gentiment Galante Garrone, était " peut-être un peu exagéré »"

13 Quinet avait été aussi parmi ses premières études révolutionnaires. Dans le cas de Buonarroti, comme on l'a vu, il reconnaissait sa dette envers Venturi, même si dès 1930, au cours de ses recherches pour sa thèse de laurea en histoire du droit constitutionnel, il était tombé sur l'opuscule anonyme Riflessi sul governo federativo applicato all'Italia, qu'il put attribuer au révolutionnaire toscan ${ }^{29}$. D'autre part, l'intérêt pour l'histoire du Risorgimento, central dans l'historiographie italienne de la période, et sollicité par le centenaire des mouvements de 1831, l'avait mené aussi à découvrir et à publier en 1931 l'opuscule d'un inconnu révolutionnaire méridional, Domenico Nicolai, Considerazioni sull'Italia $^{30}$. Comme il l'écrivait à propos d'Adolfo Omodeo et de ses études sur la culture française à l'époque de la Restauration - mais on pourrait étendre son observation à l'ensemble des historiens antifascistes -, l'étude des liens entre Risorgimento italien et " civilisation européenne » était aussi une réponse aux nationalismes, à la guerre, à la tyrannie ${ }^{31}$. Dans cette perspective, la rencontre avec la « grande révolution de la fin du 
XVIII siècle $»^{32}$, vue comme le moment essentiel de la civilisation européenne moderne, allait pratiquement de soi. Comme il le rappellera plus tard, "longtemps, trop longtemps, les historiens légitimistes, dynastiques, "modérés", provinciaux, nationalistes, fascistes, avaient raconté, avec rancune polémique, le caractère totalement étranger, d'une antithèse même entre " le Risorgimento italien et la révolution française ${ }^{33}$. Et si Venturi s'occupa de Jaurès, Mathiez et Lefebvre ${ }^{34}$, Galante Garrone se dédia à Quinet. Mais là aussi il reconnaissait simultanément une sorte de hasard dans son choix et sa dette intellectuelle envers l'historien napolitain Adolfo Omodeo, avec qui il était en correspondance épistolaire depuis 1930. Omodeo, qu'il avait finalement connu personnellement à Turin en 1931 et qui lui avait fait rencontrer Benedetto Croce, le sollicita dix ans après pour traduire La Révolution de Quinet qu'il avait longuement étudiée ${ }^{35}$.

Dans son étude sur Quinet on retrouve toujours une grande attention critique à la genèse des ouvrages qu'il prenait au fur et à mesure en considération, pour les situer dans leur temps et dans le contexte de la formation culturelle, politique et même familiale des auteurs; et toujours la même recherche acharnée de documents. Son édition italienne de La Révolution, finalement publiée en $1953^{36}$, n'était pas seulement une traduction car elle était précédée par une introduction, véritable étude critique de l'ouvrage et de son auteur, fondée sur les carnets de travail de Quinet et sur le journal de sa femme. Et comme pour "ses" autres auteurs, il y reviendra par la suite ${ }^{37}$. Dans l'œuvre de Quinet il trouvait une critique de la vision fataliste et providentielle d'autres historiens de la Révolution tels que Thierry, Thiers ou Mignet, Guizot, Louis Blanc ou Buchez et Roux. Surtout, écrivait-il, personne avant Quinet n'avait «ressenti si intensément la liberté comme centre de l'histoire ", comme «la sève de l'arbre ». À Quinet Benedetto Croce avait emprunté sa formule de la « religion de la liberté ». D'où la force mais aussi la faiblesse de l'ouvrage de Quinet, où la vie concrète des individus était presque effacée devant «des idées trop grandes». Une histoire éthique et religieuse donc, et comme l'avaient vu Adolfo Omodeo, et plus tard, François Furet, une histoire qui réfutait la vision fataliste de la Terreur qui finissait par justifier voire par exalter la Terreur elle-même, sans comprendre ou ne voulant pas comprendre qu'elle avait été une renonciation à la liberté, qu'elle avait ouvert la voie à la restauration du passé par un nouveau despotisme ${ }^{38}$. Dans l'œuvre de Quinet il retrouve aussi «son " Buonarroti au milieu des exilés, comme il écrit en 1953, la même année de sa parution en italien, dans Movimento operaio: "Il y a un chapitre, vers la fin de la Révolution d'Edgar Quinet, sur les Conventionnels en exil. Ce sont des belles pages pleines d'émotion $»^{39}$.

On peut suivre tout le long de sa production sur la Révolution française et sur le Risorgimento italien le fil conducteur que nous avons évoqué : la tension critique qui étudie le lien entre histoire et politique, entre historiographie et bataille des idées. Ainsi, après Quinet il passera à l'étude d'une des premières histoires italiennes de la Révolution française, à son avis "la seule véritable histoire de la Révolution française parue en Italie pendant tout le XIX ${ }^{e}$ siècle ", la seule capable d'en donner une vision unitaire, avant le grand ouvrage de Gaetano Salvemini ${ }^{40}$. Carlo Tivaroni (1843-1906), démocrate radical qui avait participé au Risorgimento italien en combattant comme volontaire avec Garibaldi, avait publié sa volumineuse Histoire critique de la Révolution française, en 1882, suivie par une Histoire critique du Risorgimento italien en neuf volumes (1888-1897). Dans son ouvrage, Galante Garrone retrouvait plus d'un écho de La Révolution d'Edgar Quinet, mais aussi de Thiers, Mignet, Lamartine. Il y voyait surtout 
une vision comparable à celle d'Alphonse Aulard, inscrivant tous les deux parmi les " historiens conciliateurs ", comme les avait définis Mathiez, qui essayaient de nuancer les conflits entre les révolutionnaires pour les considérer dans leur unité. La position historiographique de Tivaroni, " par son effort pour dépasser les polémiques des partis dans une supérieure vision unitaire, était donc, comme pour Aulard, avant tout le reflet d'une volonté politique $»^{41}$. Là aussi, la reconstruction attentive et documentée de la biographie politique de l'auteur lui servait à comprendre son ouvrage, à comprendre comment il était "devenu historien du Risorgimento", "par quelles suggestions et réflexions politiques» et pourquoi il avait écrit cette histoire de la Révolution française, sans laquelle il n'était pas possible de comprendre l'histoire italienne ${ }^{42}$.

On pourrait multiplier les exemples. Ainsi, dans le volume d'hommages de la Rivista storica italiana pour ses quatre-vingt ans, il écrivit un long article sur Luigi Salvatorelli en 1966, que Giuseppe Giarrizzo a considéré comme son meilleur article "autobiographique $»^{43}$, où ce qui le frappe - et qu'il partage - c'est la «vigueur de la synthèse historiographique et de la polémique actuelle $»^{44}$, et ce qu'il veut voir est comment les «idées politiques se convertissent en autant d'impulsions historiographiques $»^{45}$. Dans les ouvrages de Salvatorelli, des études du christianisme ancien jusqu'aux études sur la nation et le nationalisme et sur l'Italie durant le fascisme, il distinguait un fil rouge : la volonté de «tenir ses yeux bien ouverts sur les problèmes de son temps ", le sens "vigilant et sévère de la responsabilité des hommes dans le cours historique ", la foi inébranlable dans des valeurs inactuelles comme "la pensée, la fraternité humaine, la conscience individuelle ", la défense acharnée du Risorgimento contre toute tendance " courtisane, sanfédiste, réactionnaire " ${ }^{46}$. Comme le montrait Mazzini, la « politique nationale et populaire ne peut jamais être incarnée par des individus dominateurs, et le problème est toujours de reconduire l'Italie à l'Europe, et de concilier l'égalité avec la liberté $\aleph^{47}$. Salvatorelli avait écrit en 1961 et Galante Garrone repétait en 1966 :

« le conflit entre l'Italie des lazzaroni, des sanfédistes et des brigands et l'Italie de la civilisation européenne, la civilisation du droit, de la raison, de la liberté, est un conflit qui - rappelons-nous le bien - encore aujourd'hui n'est pas terminé »48.

Mais il y a un autre aspect essentiel de l'historiographie de Galante Garrone, bien éloigné de l'« improvisation » qu'il aimait s'attribuer : son intérêt non seulement pour le lien entre histoire et politique mais aussi et surtout pour le rapport entre individu et mouvement historique qui s'exprime par son amour de la reconstruction biographique, des personnages et des auteurs. Sur ce terrain, Gilbert Romme est sans aucun doute son chef-d'œuvre. Son modèle historiographique déclaré était Gaetano Salvemini, à la mémoire duquel l'ouvrage est dédié, en vertu de l'exhortation « à laisser de côté la soidisant Révolution pour parler en termes concrets de révolutionnaires $»^{49}$. Et en termes très concrets, l'histoire de Romme démontrait ce que Lazare Carnot avait affirmé : « On n'est pas révolutionnaire : on le devient $»^{50}$. Je ne reviendrai pas sur les contenus de cet ouvrage qui sont bien connus. Mais on peut noter encore une fois le réseau d'amitiés et de sollicitations qui entoure sa genèse : Franco Venturi, qu'il avait connu à Turin vers la fin de 1942-début 1943, l'avait mis en rapport avec Georges Lefebvre, celui-ci l'avait chargé de recherches sur les papiers Romme qui avaient abouti au Musée du Risorgimento de Milan ${ }^{51}$. C'était donc de Georges Lefebvre qu'il avait reçu «la première incitation à s'occuper de Gilbert Romme ${ }^{52}$.

Mais dans la rédaction du Romme, son modèle, comme on l'a vu, n'étaient ni Mathiez ni Lefebvre, ni Quinet ni Omodeo : c'était Salvemini qu'il avait connu en personne en 1947 
et avec qui il engagea une longue correspondance épistolaire au sujet des études sur Buonarroti, Babeuf, les saint-simoniens ${ }^{53}$. La première édition de la Révolution française de Salvemini (1905) avait été saluée en 1909 par Albert Mathiez dans les Annales révolutionnaires, comme « un excellent manuel, le meilleur sans contredit qui existe sur l'histoire de la Révolution ", même si, remarquait-il, il épousait «les aversions antirobespierristes de l'école » d'Aulard ${ }^{54}$. Il répétait ce jugement en 1926, à la parution de la cinquième édition de "cet excellent manuel ", "à l'heure même ", ajoutait-il, " où l'auteur est obligé de se réfugier en France pour échapper à la tyrannie ». Il répétait aussi ses réserves: "Bien que les préventions de M. Salvemini contre Robespierre se soient atténuées, bien que son admiration ancienne pour Danton ait à peu près disparu, je ne puis pas encore souscrire à tous ses jugements $»^{55}$.

19 On peut constater la même attitude admirative et critique à l'égard des ouvrages de Galante Garrone de la part de Georges Lefebvre dans les pages de la revue. En 1950 il fait le compte rendu du Buonarroti e Babeuf (paru en 1948). L'année précédente il avait signalé «l'excellent petit livre» de Franco Venturi sur les origines de l'Encyclopédie (1946) et rendu compte de l'ouvrage du même auteur sur Jean Jaurès (1948) ; et Jacques Godechot avait déjà rendu compte d'un article de Pia Onnis Rosa sur Filippo Buonarroti, qui remontait à 1939 mais dont les "événements" avaient "empêché de rendre compte plus tôt ${ }^{56}$. À propos du Buonarroti et Babeuf de Galante Garrone, Lefebvre soulignait l'importance des questions abordées : les relations et les différences entre les deux personnages, l'origine et les caractères de leur communisme; du crédit à accorder au témoignage tardif de Buonarroti sur la conspiration des Égaux, aux rapports avec la paysannerie et l'expérience de l'an II. Et il suggérait de développer la recherche sur la notion de dictature révolutionnaire que l'auteur avait laissée de côté : un thème sur lequel Lefebvre revenait aussi à l'occasion du IXe Congrès international des sciences historiques ${ }^{57}$.

Dans le cadre d'une attention constante, dès la fin de la guerre, à l'historiographie italienne, témoignée aussi par la publication en 1950 de l'article de Pia Onnis Rosa sur les études italiennes sur la Révolution française ${ }^{58}$, le numéro 23 de 1951 présentait ensemble les comptes rendus de Jacques Godechot et Georges Lefebvre sur les livres d'Armando Saitta et d'Alessandro Galante Garrone sur Buonarroti. En comparant les deux livres, Lefebvre rouvrait surtout la question du crédit à accorder à la Conspiration des Égaux de Buonarroti, datant de 1828, comme expression des convictions de 1796, en considération des réserves avancées par Saitta sur ce point. Mais il considérait que, " tout en tenant compte des observations critiques qui font grand honneur à M. Saitta, la conclusion de M. Garrone peut être retenue $»^{59}$. Dans ce même numéro, la Revue de la presse signalait l'article du même auteur sur les saint-simoniens et l'histoire de la Révolution française, parue en 1949 dans la Rivista storica italiana ${ }^{60}$. En 1952, comme on l'a dit plus haut, Galante Garrone entra dans le Comité de la Société des études robespierristes, dont Armando Saitta sera à son tour le vice-président à partir de 1970 : signe, encore une fois, des liens très étroits entre l'historiographie française et italienne sur la Révolution. En 1953, c'était Godechot qui s'occupait de Filippo Buonarroti e i rivoluzionari dell'ottocento (1951) et du deuxième volume du Filippo Buonarroti de Saitta :

«Les deux ouvrages - observait-il - ne font pas double emploi. D'une manière générale, on peut dire que $M$. Saitta a étudié surtout la pensée et l'action de Buonarroti proprement dites, tandis que M. Galante Garrone s'est efforcé de montrer comment les idées du conspirateur se sont transformées chez les 
révolutionnaires qui ont subi son influence. On trouvera ainsi dans les deux ouvrages tous les maillons de la chaîne qui relie Robespierre aux hommes de 1848 ».

la suite de son introduction d'extraits "jusqu'alors entièrement inédits » du journal tenu par la seconde femme de l'historien français. L'introduction, ajoutait-il, « riche en indications bibliographiques, constitue une analyse remarquable des diverses influences qui contribuèrent à former la mentalité de Quinet ». Mais son commentaire sur La Révolution de Quinet en elle-même restait très critique : se situant dans la voie ouverte par Tocqueville, il lui était «bien inférieur [...] à cause de ses préoccupations métaphysiques [...] Il ne dit mot de la liberté économique et de la lutte de classe [...] Si son livre est utile, c'est comme témoignage de l'état d'esprit qui régnait en son temps dans une partie des classes dominantes $"{ }^{62}$. Dans le même volume des Annales, Galante Garrone lui-même publiait dans les "Glanes» deux lettres de Romme tirées de la Biblioteca del Risorgimento de Milan, suivies par l'inventaire sommaire des papiers du conventionnel conservés dans la même bibliothèque, et rédigé par Jacques Godechot ${ }^{63}$. En 1955 la Revue de la presse signalait son étude sur l'émigration politique italienne du Risorgimento, parue dans la Rassegna storica del Risorgimento de $1954^{64}$ et Jacques Godechot signalait de nouveau l'article sur Filippo Buonarroti et les conventionnels en exil de 1953, en rappelant que lui-même lui avait procuré le microfilm des papiers Vadier. Galante Garrone, «que son beau livre sur Buonarroti a classé parmi les historiens qui connaissent le mieux le milieu des anciens conventionnels exilés en Belgique [...] fait précéder les lettres adressées à la famille Vadier d'une très intéresante [sic] introduction, riche de renseignements sur la vie et l'activité des exilés en Belgique »; les papiers sont suivis "de l'édition d'une des premières recensions critiques de l'œuvre de Buonarroti, « la Conspiration pour l'égalité » [...], celle qui parut dans "le Globe" du 4 avril 1829 », qui parlait de «la présence d'une doctrine absolue et désintéressée, qui, soutenue avec exaltation, fut l'âme de la conspiration la plus démocratique en principe dont nous ayons le souvenir $»^{65}$. En 1957, on signalait dans les Notices un volume de Galante Garrone (à l'époque conseiller à la Cour d'appel de Turin), Dall'Ancien régime alla Rivoluzione francese, qui aurait mérité plus de circulation et d'écho en Italie, où il représentait le recueil d'une série de leçons qui avaient été transmises par la Radio italienne en juillet 1956: un petit livre de vulgarisation donc, mais de grande valeur, fondé sur des documents et accompagné par des illustrations sur la vie quotidienne. Comme il écrivait dans sa conclusion, « du labeur de la Révolution était née non seulement une France nouvelle, mais une nouvelle condition humaine $»^{6}$. Godechot observait que le « sous-titre (Appunti per una storia del costume) inclinait à trouver dans l'ouvrage une description de la mode et qu'en effet il y eut une évolution à cet égard en rapport avec celle de la structure sociale. L'illustration procure quelques renseignements sur ce compte, mais le texte frappe surtout par le nombre des extraits tirés des publications contemporaines et des chansons de circonstance qui témoignent de la profonde connaissance de cette histoire qu'il possède ${ }^{67}$. Dans les Notices de 1957 d'ailleurs, l'Italie se voyait «remarquablement représentée parmi les tirages à part qui nous ont été envoyés $\aleph^{68}$, de Saitta à Pasquale Villani, de Carlo Zaghi à Marino Berengo, dont Godechot appréciait « une clarté, un esprit de mesure, un sens de l'évolution historique, une richesse documentaire dont on doit le féliciter et qui font honneur à l'école historique italienne $»^{69}$. 

Godechot déclarait sa «profonde émotion » dans la rédaction du compte rendu : effet, qui est à l'origine de ce livre, il a conseillé à notre ami A. Galante-Garrone d'en entreprendre la rédaction, il l'a surveillée, il en a lu les épreuves, il en a rédigé la préface dans laquelle il a posé quelques problèmes auxquels A. Galante-Garrone a répondu dans sa conclusion ».

Grâce à sa "vaste enquête, Alessandro Galante-Garrone a pu consacrer à Gilbert Romme un ouvrage dont il n'est pas exagéré de dire qu'il est à peu près définitif ». « Remarquablement écrit », il prouvait "que l'auteur est un historien confirmé qui ne veut rien avancer qui ne soit étayé de preuves solides». Il présentait en outre de nombreux extraits de documents en français, que Godechot signalait « pour ceux qui ne lisent pas l'italien $»^{70}$.

Son compte rendu du livre de Galante Garrone, en effet, Georges Lefebvre avait pu le donner juste avant de mourir, dans sa préface : même s'il n'avait pu lire, probablement, que son introduction ${ }^{71}$. Le texte original français de cette préface - dont on ne tiendra pas compte dans la traduction française de 1971 - , le dernier, peut-être, de Georges Lefebvre, fut tout de suite publié par l'Information historique ${ }^{72}$, ce qui confirme aussi la circulation immédiate du livre en France. Les réserves ne manquaient pas dans ce texte, à partir justement de la citation de Carnot: "On n'est pas révolutionnaire: on le devient ", au sujet de laquelle Lefebvre observait que le " tempérament » aussi jouait sa part. C'était un texte assez sec, qui se limitait à exprimer sa reconnaissance à Galante Garrone pour son travail d'érudition, sans entrer dans le détail de sa reconstruction qu'il n'avait probablement pas pu lire, mais dont il connaissait peut-être les grandes lignes. Il résumait donc la biographie politique de Romme, posant toute une série de questions critiques : avant la Révolution, le futur conventionnel n'avait montré « aucun penchant novateur", on ne l'avait pas vu "guerroyer contre l'Ancien Régime ", il n'avait eu une "véritable intimité » qu'avec des membres de l'aristocratie, "son tempérament ne comportait pas l'ardeur de l'homme d'action». À l'Assemblée Législative on l'avait vu "d'accord avec la majorité centriste » et à la Convention «il apparaît comme l'un de ces membres de la Plaine qui souhaitaient l'union des républicains, suivaient les Montagnards quand le salut de la Révolution leur semblait l'exiger [...], mais sans se compromettre avec la Commune et les sans-culottes [...]; il n'approuva pas la proscription des Girondins. En somme, il céda aux événements, mais sans les devancer et en conservant son indépendance ». Et comment expliquer le chemin qui l'avait conduit au calendrier décadaire et à la déchristianisation? «Il faut l'avouer: si l'on se rend compte que Romme évolua sous l'influence des circonstances, la démarche de sa pensée nous échappe en grande partie ». Dans sa conclusion, Galante Garrone répondait à ces questions soulignant encore une fois le poids des liens familiaux et amicaux, des rencontres, des événements dans l'évolution des positions d'un homme effectivement peu porté pour l'action, mais animé par une grande force morale et par une foi laïque dans la loi et dans la République. Il soulignait surtout, encore une fois, «sa juste intuition : la nécessité pour les révolutionnaires d'être unis, pour faire face aux ennemis de la Révolution » et il concluait :

«C'est le dilemme dramatique de toute révolution: comment maintenir l'unité des révolutionnaires? comment empêcher que l'autorité des lois nouvelles soit confisquée par la dictature d'une minorité, quand les sorts de la révolution, sont, ou semblent, en jeu ? $»^{73}$. 
" conciliateur ", peut-être, comme il aimera répéter encore en 1990, attentif à rejeter toute «thèse préconçue ${ }^{74}$, Galante Garrone manifestait ainsi les rapports très étroits entre l'historiographie italienne et l'historiographie française de la Révolution, qu'il avait lui-même contribué à répandre en Italie, avec Franco Venturi et Aldo Garosci surtout, dès 1949, par ses traductions chez l'éditeur Einaudi ${ }^{75}$. Mais il révélait aussi les différences entre leurs tendances respectives.

Jacques Godechot, qui était l'historien de la Révolution française le plus attentif peutêtre à la production italienne, ou du moins le plus engagé à en donner compte dans les pages des Annales, schématisait ces différences en termes d'opposition entre histoire politique et intellectuelle (dans le cas italien) et histoire économique et sociale (dans le cas français). On peut lire à ce sujet son compte rendu du «Congrès franco-italien de l'Ile d'Elbe » organisé à Portoferraio en mai 1954 par la Société toscane d'histoire du Risorgimento sur les rapports entre France et Italie à l'époque révolutionnaire et impériale, avec la participation d'une dizaine d'historiens français et une centaine d'historiens italiens, " parmi lesquels nos amis Galante Garrone, Pieri et Vaccarino de Turin, le professeur Ghisalberti de Rome, etc.». Après avoir signalé "l'intéressante communication de M. Galante Garrone sur la traduction des codes français en italien par Giovanni Gambini, d'après les papiers de ce traducteur retrouvés fortuitement à Bâle par M. Garrone », il observait, plus en général :

«Il nous est apparu que l'historiographie italienne était encore très orientée vers l'histoire politique et l'histoire intellectuelle, et ne s'attachait pas assez à l'examen des structures politiques et sociales. Nous avons essayé de montrer que le régime révolutionnaire et impérial avait entraîné la formation en Italie d'une classe nouvelle, une petite bourgeoisie de fonctionnaires et d'officiers de l'armée, qui s'est enrichie par l'achat des biens nationaux, le partage des communaux et aussi l'acquisition d'autres terres que l'abolition des fideicommis a remis dans le circuit commercial. Le professeur Nino Cortese, de Naples, a contesté cette interprétation, tandis que le professeur Dal Pane, de Bologne, a pensé - et cela nous semble pertinent - que cette classe nouvelle avait commencé à se former dans certaines régions, par exemple en Toscane, avant même la Révolution. De toutes manières, il serait désirable que des études sérieusement documentées permettent de formuler des conclusions précises [...] C'est au cours de réunions de ce genre, dont on souhaite qu'elles se multiplient, que pourra s'élaborer une collaboration internationale efficace, sans laquelle aucune grande entreprise historique ne saurait réussir $»^{76}$.

Son compte rendu rend un témoignage très intéressant sur l'importance donnée aux échanges, tout en signalant les différentes tendances à l'œuvre. L'attention à la vie économique et sociale était constamment parmi les principaux critères de jugement de Godechot sur l'historiographie italienne. Ainsi, en 1955, revenant sur la Révolution française de Salvemini à l'occasion de la parution de sa traduction anglaise, et en rappelant l'accueil reçu par ses premières éditions italiennes, il écrivait :

«Tous les historiens français furent alors unanimes à le louer comme une brillante synthèse. "Excellente synthèse, écrivait Mathiez. M. Salvemini ne s'est pas seulement inspiré des travaux les plus autorisés, il en a repensé les conclusions, et son œuvre a une valeur originale" (Annales, 1909, p. 607). Et quand la cinquième édition parut en 1926, Mathiez maintint ses appréciations, regrettant seulement que M. Salvemini n'ait pas développé son étude sur la vie chère et ses répercussions (Annales, 1926, p. 198). L'ouvrage a eu plusieurs rééditions en italien, la dernière date de 1949, mais il n'avait pas été traduit en anglais, bien que M. Salvemini, exilé pendant la dictature fasciste, eût enseigné pendant un temps à Harvard. La présente traduction, faite sur l'édition italienne de 1949, contient encore quelques 
légères modifications. La synthèse est toujours aussi brillante et quarante-sept ans après avoir été écrite, elle ne semble pas beaucoup retarder sur la science moderne : d'excellents chapitres sur les classes sociales, sur le mouvement intellectuel, sur la chute du régime féodal. Il n'y manque, comme Mathiez le regrettait en 1926, qu'une étude sur la vie économique, et surtout sur la crise de 1787-89: M. Salvemini n'a pas tenu compte des travaux de M. Labrousse. Il est regrettable, en outre, que la courte note bibliographique renvoie le lecteur à la seule collection Clio : il eut été utile de citer la dernière édition du livre de $\mathrm{M}$. Lefebvre sur la Révolution ${ }^{77}$.

\section{l'intérêt des historiens italiens de son époque pour la Révolution française : \\ «Ce fut justement la réaction morale et politique au fascisme et à ses exaspérations totalitaires et nationalistes, qui fit sentir à quelques-uns de nos historiens le besoin de rechercher les liens entre notre Risorgimento et l'histoire européenne, et, par cette voie, de se rapprocher de la grande Révolution ".}

31 Il rappelait le rôle joué par Adolfo Omodeo qui, "à la suite d'une suggestion qui lui avait été faite par Benedetto Croce », l'avait poussé à traduire Quinet. Un choix, ajoutait-il, qui avait surpris Georges Lefebvre, qui lui avait manifesté «sa surprise » qu'on publiât en Italie un ouvrage désormais si pauvre d'intérêt pour l'« histoire positive » :

«Il ne pouvait se rendre compte que, chez nous, le fascisme avait créé la nécessité de recommencer derechef, de remonter aux sources libérales et démocratiques du $\mathrm{XIX}^{\mathrm{e}}$ siècle, de comprendre - dans sa valeur éternelle et dans ses limites contingentes, - ce que Quinet et Croce avaient appelé la “ religion de la liberté ". Il trouvait aussi un peu bizarre que chez nous, sous l'influence de Croce, se manifestât un goût si vif et si persistant pour l'histoire de l'historiographie $»^{78}$.

D'où l'engagement des historiens italiens, en particulier de Franco Venturi, à connaître et faire connaître les acquisitions les plus récentes de l'historiographie française de la Révolution, puisque, sauf «quelques cas isolés, on s'était arrêté, tout au plus, à Aulard ». Le " petit livre» de Venturi sur Jean Jaurès, Mathiez, Lefebvre, avait été " pour bien des Italiens, une authentique révélation » et avait éclairé « la personnalité d'historien de Georges Lefebvre [...] dans ses aspects les plus importants " ${ }^{79}$. Grâce à Venturi, et aux traductions italiennes de Quatre-vingt-neuf, des Thermidoriens, du Directoire et de La Grande Peur, Lefebvre, qui «ne se faisait aucune illusion sur le nombre, en France et ailleurs, de ses disciples et de ses partisans », trouva en Italie «bien plus qu'il ne s'y attendait, surtout parmi les plus jeunes, des lecteurs attentifs et pleins de sympathie ", parmi lesquels il rappelait surtout Rosario Romeo, selon lequel «les jugements de l'historien français avaient une importance qui allait au-delà de l'histoire de la Révolution même ", et qui avait su profiter " des intuitions historiques de Georges Lefebvre, dans son excellente étude sur "Risorgimento e capitalismo" ». Armando Saitta, de son côté, dans son introduction au recueil d'études Sanculotti $e$ contadini (1958), parmi lesquels Foules révolutionnaires, avait souligné dans les œuvres de Lefebvre «la parfaite fusion de l'histoire politique avec l'histoire économique et sociale", tout en relevant «certaines limites" de ses "considérations méthodologiques». Il rappelait enfin son attention à la production italienne - 
"Georges Lefebvre lisait couramment l'italien" - et ses nombreux comptes rendus dans les Annales ${ }^{80}$. C'est un autre témoignage significatif des échanges entre les deux historiographies, d'un rapport fondé sur des « sentiments non seulement d'admiration mais d'affectueuse et reconnaissante amitié ${ }^{81}$, mais aussi sur la critique respectueuse et féconde.

De Babeuf à Buonarroti et Romme, Galante Garrone essaya de suivre les parcours de l'idée d'égalité et de ses implications sociales dans l'histoire du XIX ${ }^{e}$ siècle, la force nouvelle et le nouveau sens qu'elle avait acquis par la Révolution française. Il sentait de partager cet attrait avec ses anciens compagnons de route, de pensée et d'action: Franco Venturi, qui dans son Settecento riformatore (1984), avait affirmé que l'égalité était peut-être la plus vivante des passions et des idées qui « éclairaient et animaient la crise suprême de l'Ancien Régime" ; Arturo Carlo Jemolo, qui le 6 octobre 1951, lui avait écrit qu'on ne se rendait peut-être pas bien compte « que ce qui a caractérisé les derniers cent-cinquante ans a été l'ébranlement continu de toute idée de privilège, la pénétration de cette religion de l'égalité qui est l'essence du christianisme, même si elle n'a pas toujours été, et au contraire l'a été plutôt rarement affirmée sous l'enseigne de la croix $»^{82}$.

Cet historien "improvisé», comme il se considérait, garda toujours un goût inépuisable pour la recherche documentaire et un sens très vif des différents contextes historiques qu'il empruntait à Tocqueville, trop souvent cité, observait-il, "par les fauteurs de la continuité ", alors qu'il avait ressenti le besoin, d'un côté, de remonter à l'Ancien Régime pour comprendre la Révolution et l'Empire, et de l'autre, d'encadrer le cas français dans une analyse comparative plus large de l'espace européen ${ }^{83}$; et à Georges Lefebvre qui, dans son Napoléon, incitait à considérer les institutions napoléoniennes dans leurs spécificités et dans leurs différences d'un pays à l'autre, leur " configuration dans l'espace et dans le temps " $^{84}$. Prêt aussi à prendre ses distances avec Georges Lefebvre quand celui-ci datait, trop rapidement à son avis, l'entrée du socialisme "dans l'histoire politique » dès la conspiration des Égaux : «En histoire, à chaque jour son labeur $»^{85}$.

\section{NOTES}

1.Alessandro Galante Garrone. Commemorazione tenuta il 25 marzo 2004, Accademia delle Scienze di Torino, s.d. [2004], avec les contributions de Giuseppe Galasso, Franzo Grande Stevens, Umberto Levra et Massimo L. Salvadori, dont celles de Franzo Grande Stevens, sous le titre " Alessandro Galante Garrone », et d' Umberto Levra, sous le titre "L'albero della libertà di Sandro Galante Garrone », ont été publiées aussi dans la Rassegna storica del Risorgimento, XCI, I, janvier-mars 2004, p. 59-67, 68-75. Le Bollettino storico-bibliografico subalpino, CII, 2004, a publié la nécrologie rédigée par Gian Paolo Romagnani, « Alessandro Galante Garrone », p. 605-609. D'autres contributions ont été publiées dans les Actes du colloque tenu le 17 mars 2004 à Vercelli, I Galante Garrone : Una famiglia vercellese del Novecento, a cura di Edoardo Tortarolo, Vercelli, Università del 
Piemonte Orientale A. Avogadro e Comune di Vercelli, 2004, avec le discours tenu le 24 avril 2001 par Edoardo Tortarolo à l'occasion de l'attribution de la citoyenneté honoraire : Edoardo Tortarolo, « Per Alessandro Galante Garrone », p. 7-10 ; Gian Paolo Romagnani, « Alessandro e Carlo Galante Garrone : tra storiografia, politica, e impegno civile », p. 11-21; Alberto Cavaglion, « Tra Salvemini e Mazzini (con una postilla su Mario e l'incantatore di Thomas Mann)», p. 23-28, et la contribution sur sa sœur, Virginia Galante Garrone, de Giusi Baldissone, « Gli archivi danteschi di Virginia ", p. 29-39. Le 10 décembre 2004, Giuseppe Giarrizzo a lu à l'Académie des Lincei son texte Per Alessandro Galante Garrone (1909-2003).

2. Alessandro GALANTE GARRONE, Il mite giacobino. Conversazione su libertà e democrazia raccolta da Paolo Borgna, Roma, Donzelli, 1994, p. 29-31.

3.Ibid., p. 111.

4. « Prefazione » à Adolfo OMODEO, Studi sull'età della Restaurazione, Torino, Einaudi, 1970 et 1974, p. XXIV ; voir aussi Alessandro GALANTE GARRONE, I miei maggiori, Milano, Garzanti, 1984, p. 81; Id., L'albero della libertà. Dai giacobini a Garibaldi, Firenze, Le Monnier, 1987, p. XIII-XIV ; Id., Libertà liberatrice, supplément à La Stampa, Torino, 14 octobre 1992. Sur ce « concept clé » de son « esprit civique », cf. aussi SALVADORI, op. cit., p. 28-29.

5.GALANTE GARRONE, Il mite giacobino, op. cit., p. 41.

6.Lettre citée par Grande STEVENS, Alessandro Galante Garrone, op. cit., p. 60-61.

7.Procès-verbal de l'Assemblée générale de la Société du 27 avril 1952, présidée par Georges Lefebvre, dans Annales historiques de la Révolution française, vol. 24, 1952, p. 442-443.

8.Je tiens à remercier M. le Conservateur Alain Chevalier qui par son accueil m'a permis de conduire cette recherche dans les Bibliothèques Soboul, Godechot et Suratteau du Centre de documentation historique de Vizille.

9.Alessandro GALANTE GARRONE, Gilbert Romme. Storia di un rivoluzionario, Prefazione di Georges Lefebvre, Torino, Einaudi, 1959 ; Gilbert Romme. Histoire d'un révolutionnaire (1750-1795), traduit de l'italien par Anne et Claude Manceron, préface de Georges Lefevbre, Paris, Flammarion (L'Histoire), 1971.

10.Gilbert Romme (1750-1795), Actes du colloque de Riom (19 et 20 mai 1995), Textes réunis et présentés par Jean EHRARD, Paris, Société des Amis du Centre de Recherches Révolutionnaires et Romantiques, Société des Études Robespierristes, 1996 ; reproduit aussi dans les Annales historiques de la Révolution française, n 304, avril-juin 1996 (d'où je cite).

11.Jean EHRARD, « Avant-propos », ibid., p. 191-192.

12.Ibid., p. 448.

13.Ibid., p. 457.

14. "Gilbert Romme et les débuts de la Société des “ Amis de la Loi " et « Gilbert Romme et les jacobins aux débuts de la Législative », dans Gilbert Romme (1750-1795) et son temps, actes du colloque tenu à Riom et Clermont les 10 et 11 juin 1965, avant-propos de Jean Ehrard et Albert Soboul, Faculté des Lettres et Sciences humaines de l'Université de Clermont-Ferrand, Publications de l'Institut d'Études du Massif-Central, fascicule I, Paris, Presses Universitaires de France, 1966, p. 93-107, 109-111.

15.Alessandro GALANTE GARRONE, Filippo Buonarroti e i rivoluzionari dell'Ottocento (1828-1837), Torino, Einaudi, 1972, première édition 1951; Philippe Buonarroti et les révolutionnaires du XIX ${ }^{\mathrm{e}}$ siècle (1828-1837), traduit de l'italien par Anne et Claude Manceron, Paris, Éditions Champ Libre, 1975. 
16.Ibid., p. 9.

17.Armando SAITTA, Filippo Buonarroti. Contributo alla storia della sua vita e del suo pensiero, 2 vol., Roma, Edizioni di Storia e Letteratura, 1950-1951, 2e éd. 1972.

18.Alessandro GALANTE GARRONE, Buonarroti e Babeuf, Torino, De Silva, 1948.

19.Alessandro GALANTE GARRONE, « Filippo Buonarroti e i Convenzionali in esilio (Dalle carte inedite della famiglia Vadier) », dans Movimento operaio, V, 3, mai-juin 1953, p. 3-65 : p. 5, note 5. L'article fut signalé dans les « Notices» et dans la revue de la presse des Annales historiques de la Révolution française, 26, 1954, p. 275 et 377, en rappelant que Jacques Godechot avait donné note des papiers Vadier dans le ${ }^{\circ} 4$ de l'année précédente.

20.GALANTE GARRONE, « Filippo Buonarroti e i Convenzionali in esilio », op. cit., p. 6, note 9, p. 23, note 42 .

21. Comme le rappelle GALANTE GARRONE, «Filippo Buonarroti », dans L'albero della Rivoluzione. Le interpretazioni della Rivoluzione francese, a cura di Bruno Bongiovanni e Luciano Guerci, Torino, Einaudi, 1989, p. 84-89: 85.

22.Alessandro GALANTE GARRONE, « Da Pratile alla cospirazione degli Eguali (Romme, Goujon, Tissot, Buonarroti) ", dans L'età dei Lumi. Studi storici sul Settecento europeo in onore di Franco Venturi, Napoli, Jovene, 1985, I, p. 395-452 : 399, note 3.

23. "Filippo Buonarroti ", dans L'albero della Rivoluzione, op. cit.

24.Filippo Buonarroti, La Riforma dell'Alcorano, a cura di Alessandro Galante Garrone e Franco Venturi, Palermo, Sellerio, 1992.

25.GAlANTE GARRONE, « Da Pratile alla cospirazione degli Eguali », op. cit., p. 411, note 18. 26.Ibid., p. 399, note 3 (points de suspension dans le texte). Il ajoutait avec sa modestie usuelle : «Et les signes de cette improvisation n'ont pas encore disparu ». Sur le rôle de Venturi dans ses études sur Buonarroti, voir aussi, plus largement, son introduction à Franco VENTURI, Scritti politici. La lotta per la libertà, Saggi introduttivi di Vittorio Foa e Alessandro Galante Garrone, a cura di Leonardo Casalino, Torino, Einaudi, 1996, pp. XXV-LI, et sa « Testimonianza su Franco Venturi », dans Il coraggio della ragione. Franco Venturi intellettuale e storico cosmopolita, a cura di Luciano Guerci e Giuseppe Ricuperati, Torino, Fondazione Luigi Einaudi, 1998, p. 415-424.

27. "Filippo Buonarroti », dans L'albero della Rivoluzione, op. cit., p. 86. Sur le rapport Galante Garrone-Albert Mathiez, comportant l'admiration pour ses études et son « sens historique » et en même temps la distance par rapport à son attitude « philorobespierriste », à son « robespierrisme trop accentué » et à son « interprétation buonarrotienne de la révolution française », cf. GALASSO, « Commemorazione », op. cit., p. 5-8.

28. " Edgar Quinet », dans L'albero della Rivoluzione, op. cit., p. 535-542 : 535.

29.GALANTE GARRONE, « Testimonianza su Franco Venturi », op. cit., p. 416-417.

30.Cf. GALANTE GARRONE, L'albero della libertà, op. cit., p. VII et passim.

31.GALANTE GARRONE, « Prefazione » à Omodeo, op. cit., p. XII-XVI.

32.Ibid., p. XVII.

33.Alessandro GALANTE GARRONE, « La Rivoluzione francese e il Risorgimento italiano », dans L'eredità della Rivoluzione francese, a cura di François Furet, con la collaborazione di Massimo Boffa, Roma-Bari, Laterza, 1989, p. 159-196 : 159.

34.Franco VENTURI, Jean Jaurès e altri storici della Rivoluzione francese, Torino, Einaudi, 1948. 
35.Sur ses rapports avec Omodeo et la genèse de sa traduction de Quinet, cf. GALANTE GARRONE, L'albero della libertà, op. cit., p. VI-IX et sa préface a Isa Angrisani Guerrini, Quinet e l'Italia, Genève-Paris, Slatkine, 1981, reproduite sous le titre « Quinet e l'Italia », ibid., p. 251-266 : 252.

36.Edgar QUINET, La rivoluzione, Torino, Einaudi, 1953, 2 vol.

37.En 1981 avec « Quinet et l'Italie », op. cit.; en 1989 avec l'article « Edgar Quinet », op. cit.

38.Ibid., p. 538.

39. GALANTE GARRone, Filippo Buonarroti e i Convenzionali in esilio, op. cit., p. 3.

40.Alessandro GALANTE GARRONE, «Carlo Tivaroni : come divenne storico del Risorgimento », dans Rivista storica italiana, LXXIX, 1967, p. 313-354 : 339.

41.Ibid., p. 313-314.

42.Ibid., p. 339. Voir aussi Alessandro GALANTE GARRONE, « Carlo Tivaroni », dans L'albero della Rivoluzione, op. cit., p. 629-633.

43. GIARRIZZo, Per Alessandro Galante Garrone, op. cit.

44.Alessandro GALANTE GARRONE, « Risorgimento e antirisorgimento negli scritti di Luigi Salvatorelli », dans Rivista storica italiana, LXXVIII, 1966, p. 513-543 : 513.

45.Ibid., p. 525.

46.Ibid., p. 515, 519, 524, 513.

47.Ibid., p. 534.

48.Ibid., p. 543.

49.GALANTE GARRONE, Gilbert Romme. Storia di un rivoluzionario, op. cit., p. 15.

50.Ibid., p. 14.

51.gAlante GARRONe, Testimonianza su Franco Venturi, op. cit., p. 423.

52.GALANTE GARRONE, Gilbert Romme. Storia di un rivoluzionario, op. cit., p. 15.

53.Alessandro GALANTE GARRONE, Salvemini e Mazzini, Messina-Firenze, D’Anna, 1981, p. 9-15.

54.Annales révolutionnaires, II, 1909, p. 607-608.

55.Annales historiques de la révolution française, n.s., III, 1926, p. 198.

56.Ibid., 21, 1949, p. 281-282, 373-374, 374-376.

57.Ibid., 22, 1950, p. 78-82. Dans le même numéro on signalait, dans la revue de la presse, l'article de Galante Garrone sur les premiers jacobins piémontais, paru dans Il Ponte, août 1949 (ibid., p. 95). Sur Lefebvre et le babouvisme au IXe Congrès international des sciences historiques, cf. ibid., 25, 1953, p. 82, compte rendu de Godechot sur Galante Garrone.

58.Pia ONNIS ROSA, « Les études italiennes sur l'histoire de la Révolution française de 1940 à $1949 »$, ibid., p. 358-361.

59.Ibid, 23, 1951, p. 89-94.

60.Ibid., p. 111.

61.Ibid., 25, 1953, p. 80-84: 80, 82.

62.Ibid., 26, 1954, p. 182-183.

63.Ibid., p. 77-82.

64.Ibid., 27, 1955, p. 398-399. L'article, qui était le texte du rapport présenté au Congrès d'histoire du Risorgimento de 1953, est reproduit dans GALANTE GARRONE, L'albero della libertà, op. cit., p. 59-97.

65.Annales historiques de la Révolution française, 27, 1955, p. 93. 
66. Alessandro GALANTE GARRONE, Dall'Ancien régime alla Rivoluzione francese. Appunti per una storia del costume, Torino, Eri, 1956, p. 177. Il y en a deux exemplaires dans le Centre de documentation de Vizille, l'un envoyé à Soboul, l'autre à Godechot.

67.Annales historiques de la Révolution française, 29, 1957, p. 282-283.

68.Ibid., p. 86-87.

69.Ibid., p. 370-373. De même, dans le vol. 31, 1959, Godechot répétait, à propos du « livre intelligent, perspicace et mesuré » de Gaetano CINGARI, Giacobini e sanfedisti (1957) : « Voilà encore une œuvre qui fait honneur à la jeune école historique italienne ».

70.Annales historiques de la Révolution française, 32, 1960, p. 335-339.

71.En conclusion de son hommage à Lefebvre, en effet, Galante Garrone observait : « Un des derniers écrits de Georges Lefebvre, peut-être le dernier qui ait paru de son vivant, est la préface à la biographie que j'ai publiée en août 1959 [...]. Je ne le saurai sans doute jamais : mais j'aime à croire que ses yeux ont encore pu voir ce livre, qui lui doit beaucoup » ("Georges Lefebvre et les historiens italiens », dans Annales historiques de la Révolution française, 32, 1960, p. 116).

72.Information historique, 1960, n. 3, p. 118-119 : ce texte est inséré dans la copie du Romme de Galante Garrone d'Albert Soboul, qui signale sa traduction dans une note à côté de la Préface italienne.

73. GALANTE GARRONE, Gilbert Romme, op. cit., p. 510-511.

74.Alessandro GALANTE GARRONE, « All'ombra dell'aquila imperiale », dans All'ombra dell'aquila imperiale. Trasformazioni e continuità istituzionali nei territori sabaudi in età napoleonica (1802-1804), Atti del convegno, Torino 15-18 ottobre 1990, Roma-Torino, Ministero per i beni culturali e ambientali, Ufficio Centrale per i beni archivistici, Pubblicazioni degli Archivi di Stato, 28, 1994, I, p. 18-25 : 25.

75. Georges LEFEBVRE, L'Ottantanove, Traduzione e Introduzione di Alessandro Galante Garrone, Torino, Einaudi, 1949 ; Id., La grande paura del 1789, a cura di Aldo Garosci, Torino, Einaudi, 1953.

76.Annales historiques de la Révolution française, 26, 1954, p. 279-280.

77.Ibid., 27, 1955, p. 82.

78. GALANTE GARRONE, « Georges Lefebvre », op. cit., p. 109-110.

79.Ibid., p. 110-111.

80.Ibid., p. 114-116.

81.Ibid., p. 115.

82. GALANTE GARRONe, Da Pratile alla cospirazione degli Eguali, op. cit., p. 443, note 42.

83.GALANTE GARRONE, « All'ombra dell'aquila imperiale », op. cit., p. 18-20.

84.Ibid., p. 22.

85.GAlANTE GARRone, Da Pratile alla cospirazione degli Eguali, op. cit., p. 452. 